\title{
Adopting an Agile Approach for the Development of Mobile Applications
}

\author{
Harleen K. Flora \\ The IIS University \\ Jaipur, India
}

\author{
Swati V. Chande \\ International School of \\ Informatics and Management \\ Jaipur, India
}

\author{
Xiaofeng Wang \\ Free University of \\ Bozen/Bolzano, Italy
}

\begin{abstract}
With the increasing popularity and demand for mobile applications, there has been a significant increase in the number of mobile application development projects. Highly volatile requirements of mobile applications require adaptive software development methods. The Agile approach is seen as a natural fit for mobile application and there is a need to explore various Agile methodologies for the development of mobile applications. This paper evaluates how adopting various Agile approaches improves the development of mobile applications and if they can be used in order to provide more tailor-made process improvements within an organization. A survey related to mobile application development process improvement was developed. The use of various Agile approaches for success in mobile application development were evaluated by determining the significance of the most used Agile engineering paradigms such as XP, Scrum, and Lean. The findings of the study show that these Agile methods have the potential to help deliver enhanced speed and quality for mobile application development.
\end{abstract}

\section{Keywords}

Software Engineering, Mobile Computing, Mobile Application Development, Agile Methodology, Scrum, XP, Lean

\section{INTRODUCTION}

\subsection{Mobile Software Development}

In recent years, the mobile industry has witnessed rapid expansion and the potential number of different mobile applications is almost unlimited. With the increasing popularity and demand for mobile applications, there has been a significant increase in number of projects for mobile application development services. As a result of this, the quality and quantity of the mobile applications has introduced new concerns in computer science and industry.

Mobile software development is the process of creating software which can be used by small, low-power handheld devices such as mobile phones. This software is either factory pre-installed applications on mobile phones or downloadable from app stores and mobile software distribution platforms. The development of mobile applications is challenging due to rapidly changing business requirements and technical constraints associated with mobile systems. Development teams face the challenge of a dynamic environment with frequent modifications in customer needs and expectations.
The rapid growth of mobile computing platform has almost outpaced the software engineering processes tailored to mobile application development with significant modifications. However, at the moment, there is limited knowledge about the suitability of different software practices for the development of mobile applications. Agile software development practice have caught the attention of software development teams and software engineering researchers worldwide during the last decade but scientific research and published outcomes still remains quite scarce.

\subsection{Agile Software Development}

Several software development methodologies began to attract public attention in the late 1990's. In February 2001, 17 software developers met to discuss lightweight development methods and published the Manifesto for Agile Software Development, designed to find better ways of developing softwares. The Agile approach values, "Individuals and interactions over processes and tools, Working software over comprehensive documentation, Customer collaboration over contract negotiation, and Responding to change over following a plan" [1].

Agile methodologies follow an iterative approach to build software rapidly where the entire software development lifecycle is broken down into smaller iterations which helps to minimize the overall risk, allows the project to adapt to modifications rapidly, does not demand a requirements freeze upfront, and maintains the project schedule and budget. The Agile approach to software development refers to the iterative and incremental strategy involving self-organizing teams and cross-functioning teams working collaboratively to create software. Some of the well-known existing agile software development methods are XP, Scrum, FDD, DSDM, Kanban, ASD, AUP, AM, Lean and Crystal.

This paper investigates new research directions on mobile applications development using Agile approaches by answering the following research questions: a) What are reasons of adopting Agile approaches for the development of mobile applications? b) What are an organizations greatest concerns regarding adoption of Agile for mobile projects, leading causes of failed mobile projects using Agile, and barriers to further adopt Agile practices for mobile projects? c) Which Agile approach (or combination) is most appropriate and most closely followed for the development of mobile applications and why? The study presents the result of extensive survey conducted to determine suitable Agile practices currently being used for the development of mobile applications. 


\section{LITERATURE REVIEW}

This section provides a review of the existing scientific literature on mobile software development using Agile methodologies. Like in traditional software projects, there are number of major parts in the end-to-end process of developing a mobile application in order to be successful. Studies have identified unique challenges for mobile application development, such as form factors and user input technology, usability and user interaction design, choice of implementation technology for mobile app implementation, issues based upon development processes, tools, user interface design, application portability, quality, and security [2].

\subsection{Agile - A natural fit for mobile software}

To provide a solution to the above discussed challenges, it has been proposed that Agile is a natural fit for software development for mobile devices $[3,4,5,6,7,8]$ and studies carried out for the application of Agile development approaches to mobile application development indicate the need for software development processes tailored to suite the mobile application requirements. Research $[9,10]$ shows that Agile methodologies are one of the best practices to be adhered for mobile software development process.

As per Abrahamsson [11], Agile themes can be mapped to the traits observed in mobile software development and Agile fits mobile software development well. For example, Scrum is focused on project management and this methodology has natural advantages in development of mobile applications based on having a disciplined and limited scope, high end user interaction, and a condensed time-to-market. Mobile companies and even individual developers are following a Scrum-like process as they develop mobile applications [12, $13,14,15]$.

Though Scrum is the most popular Agile method and the most mature Agile teams use Scrum as their project management framework. However, it says very little about the engineering discipline required to deliver high quality products. XP provides 12 practices and other values that can reenergize team and help guarantee high quality software. It is interesting to note that mixing practices from Scrum and XP hybrid has shown significant results in software development projects, specifically for mobile devices [16].

\subsection{Proposed Agile practices for the development of mobile software}

To understand on how Agile practices can be implemented in a mobile project, we surveyed research papers on mobile application development using Agile methods. As a result, we found four Agile approaches, some with the combination of non-Agile approaches: Mobile-D, HME, MASAM, and SLeSS as discussed below.

Mobile-D: One of the pioneering studies in Agile approach is by Abrahamsson et al. [17]. It shows that Agile development provides a good fit for mobile application development environment and proposes an approach called Mobile D. It is based on XP, Crystal methodologies and Rational Unified Process (RUP) and is recommended to be used by small, colocated teams working on short development cycles. MobileD comprises five phases (Explore, Initialize, Productionize, Stabilize and System Test and Fix), each of which has a number of associated stages, tasks, and practices. Though Mobile D for mobile software development seems to be focused and promising, the description provided for this approach is brief and incomplete, some of its techniques, such as Architecture Line may not be adopted.

HME: Rahimian et al. [18] proposed a new Agile approach, the Hybrid Method Engineering (HME), created from Methodology Engineering techniques. It is based on a combination of Agile methodologies, ASD and New Product Development (NPD). It was developed in four iterations: in the first iteration, the methodology was detailed by adding practices commonly found in Agile methods; taking into account market considerations, the second iteration included activities from NPD, a process concerned with introducing a new product or service to the market; in the third iteration, ASD ideas were integrated into the methodology, while in the final iteration prototyping was added to mitigate likely technology-related risks. Though HME is mobile application development focused but it is a high-level abstraction and does not provide its phases with the details needed to apply it to customized development for mobile phones.

MASAM: Jeong et al. [19] proposed MASAM (Mobile Application Software Agile Methodology) that provides the process for developing the application software operated on mobile platform. It is based on XP, AUP, RUP, and the software and systems process engineering meta-model. It proposes a simple development cycle with four phases: Preparation Phase, that defines a summary and a first notion of the product, and assigns roles and responsibilities; Embodiment Phase, that focuses on understanding users' needs and defines the architecture of the software product; Product Developing Phase, that follows the principles of XP including test driven development, pair programming, refactoring and continuous integration with a close relationship with iterative testing activities; and Commercialization Phase, that concentrates on product launch and product sales. However, this approach is unclear, does not include a case study and is cited by few papers but none of them reports an experience of its utilization.

SLeSS: Cunha et al. [20] proposed SLeSS, an integration approach of Scrum and Lean Six Sigma used in real projects of developing embedded software customizations for mobile phones. This approach enables the achievement of performance and quality targets, progressively improving the development process and the outcome of projects. This paper includes a case study of an implementation in a mobile software company, but there is no report of its utilization.

Based upon literature review, it was found that there is no research study that provides detail reasons on why Agile as most suitable for mobile application development, what are organizations greatest concerns regarding Agile adoption, leading causes of failed and barriers to further adopt Agile practices for mobile projects. Also, no studies were found addressing Scrum, XP and Lean integration for software development for mobile phones and, for that reason, this article aims to encourage and contribute to such a discussion.

\section{RESEARCH APPROACH}

An extensive survey was conducted for this study to gain a better understanding of suitable Agile approaches currently practiced by mobile companies for the development of mobile applications. An online questionnaire was designed based on the available literature. In addition, the design of the 
questionnaire was evaluated and validated by several experts in Agile and mobile development.

The main contributors to the survey were the mobile community, including development team members, Agile experts, researchers, and other stakeholders. They were invited to participate in the survey via mobile developer forums which covered multiple aspects of mobile application development. More than 130 responses were received and the participation in the survey was entirely voluntary.

The data was analysed quantitatively and qualitatively by studying each response individually and then analysing all responses collectively. The validity of the study and findings were assessed by experts in respective areas. Since the focus of this paper is on examining the suitability of Agile practices for the development of mobile applications, we report the part of the survey results that are pertinent to this focus. The results of the survey were utilized as a starting point to identify specific demands for using Agile for mobile software engineering.

Figures 1 to 7 show general information about the participants. Figures 1-3 are about the organizations, Figures 4-5 are about the mobile apps developed in these organizations and Figures 6,7 are about the individual respondents. The values in each figure is depicted in percentage.

It can be seen from the figures that $69 \%$ of respondents came from the mobile industry and had actual mobile application development experience.

Most of the respondents had experience in developing mobile software, mainly in education (14\%), utilities (14\%) and communication $(13 \%)$.

Maximum response was received from development team members $(47 \%)$ as compared to consultants/trainers (33\%) and top level management (20\%).

Respondents came from a variety of locations, with the biggest group (38\%) coming from North America.

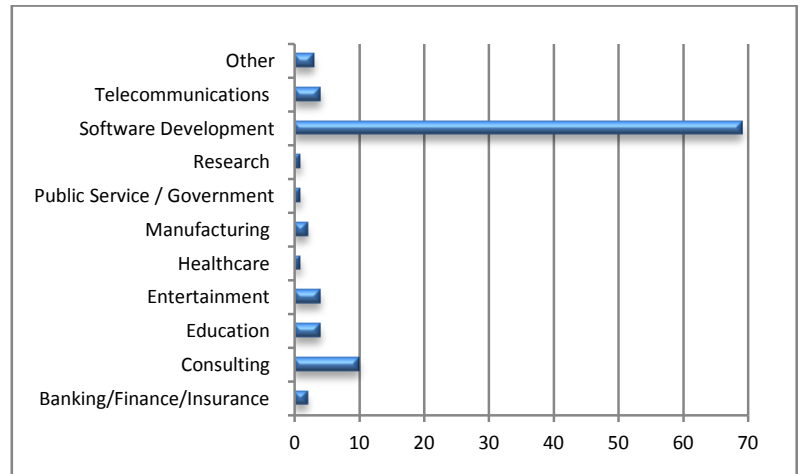

Figure 1: Organization Type.

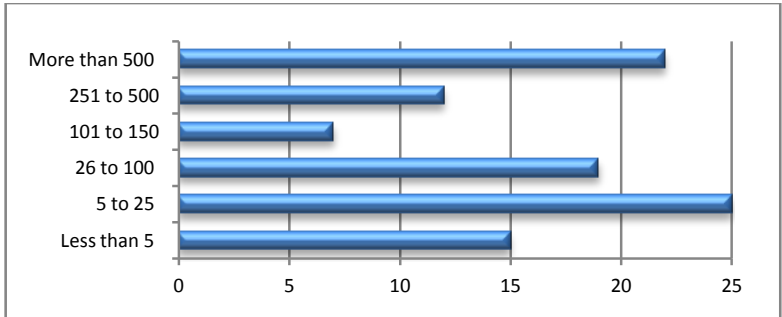

Figure 2: Organization Size

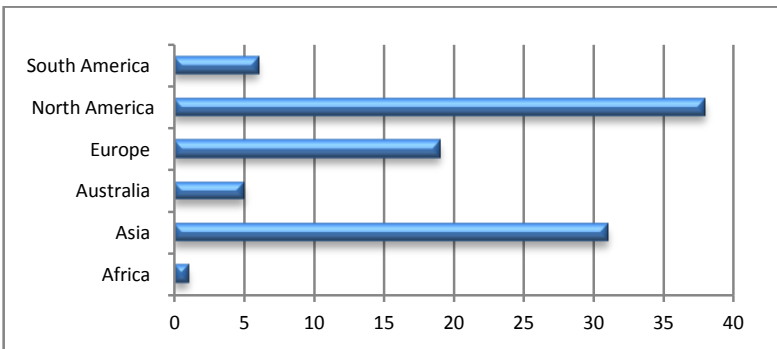

Figure 3: Organization Location

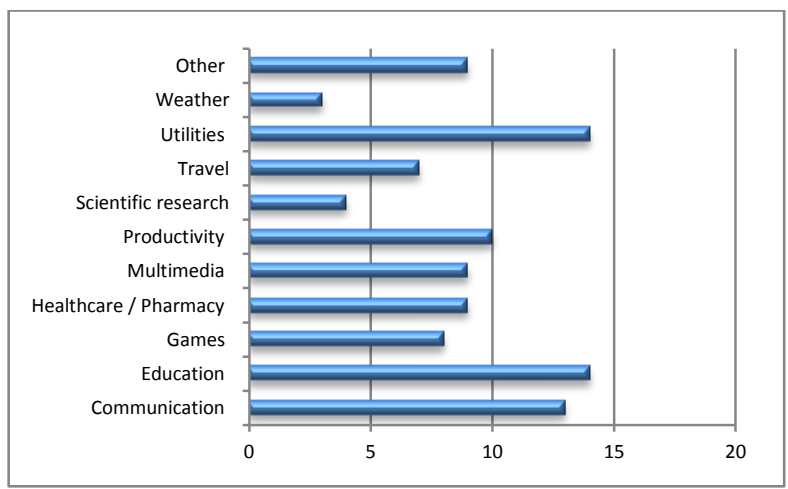

Figure 4: Categories of Mobile Applications Developed.

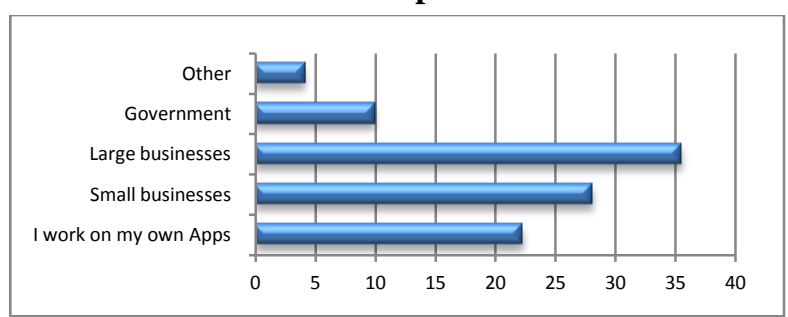

Figure 5: Who commissions the app.

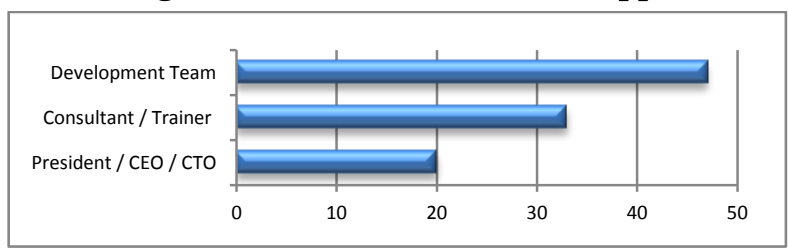

Figure 6: Respondent Role in Organization.

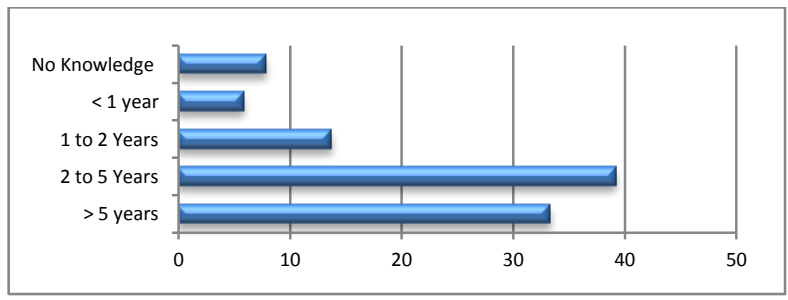

Figure 7: Agile development methods practicing experience. 


\section{FINDINGS}

\subsection{Regarding the suitability of Agile approach for mobile projects}

Agile processes were considered to be very appropriate with software for fast-paced markets, where customer satisfaction is governed by early and frequent delivery, where there is scope for changes even late in the project, the delivery cycle is short (e.g. every couple of weeks), there is appropriate collaboration between businesses and developers, where working software is the primary measure of progress, and where is there continuous attention to technical excellence and good design and simplicity.

$86 \%$ of survey participants believed that Agile methods and practices are appropriate for the development of mobile applications. The key advantage for using Agile methodologies for mobile applications is that iterative development helps keep focus on the consumer market (28\%), quick release to meet market demand (24\%), ease of fixing the issues for each version (20\%), the ability to manage development with a geographically distributed team (15\%), and $13 \%$ other reasons. These other reasons included faster time to market, better quality, release before major OS upgrade, realism, assurance of building the right thing the right way, fast feedback based on tangible results, and short delivery cycles.

Regarding the suitability of Agile approaches for mobile application development, participants were asked two questions: 1) Do you believe that Agile methods and practices are appropriate for mobile application development and why? 2) What advantages / values were realized from implementing Agile practices in mobile application development? It was found that Agile development methods are a natural fit for mobile apps and the following are significant ways in which Agile practices enhances and speeds up the development of mobile app projects:

Agile works well with highly volatile requirements of mobile apps: Highly volatile requirements of mobile apps require adaptive software development methods. Agile is ideal for projects with high levels of uncertainty or variability and are appropriate for mobile app development. Agile enables easy accommodating of change requests, frequent interaction with clients, and helps in unifying the business requirements across teams by assigning the right priorities and focuses. Agile is most suitable to address the issues related to inadequate memory; data storage capability; slower devices; security; online/offline capability; and ever-evolving types of devices, operating systems, and development environments.

Agile development encourages stakeholder involvement in mobile projects: Stakeholder engagement in mobile projects enables monitoring of activities which improves increases productivity, profit, and sustainability. Agile development principles encourage active user involvement throughout product development and a very cooperative collaborative environment. This provides excellent visibility for key stakeholders, enables rapid accommodation of stakeholder feedback, and helps in rapidly rolling out suggested new features, and ensuring that expectations are effectively managed.

Agile increases reliability and leads to continued use of mobile apps: Mobile users are usually less tolerant of errors and crashes in mobile apps. If an app crashes a few times, the mobile user will easily switch an alternative app as they have many choices available. Agile development with its iterative testing and quality assurance practices assists developers to build in more quality and reliability through repeated cycles of testing.

Agile development empowers user experience for mobile apps: Mobile apps run in limited environments and there are restrictions when downloading apps. If downloading an app takes several minutes, the user may try other apps instead. Agile development enables thoughtful user experiences and allows developers to experiment different options in subsequent sprints and adjust the design and features of apps to make the user experience is quick, smooth, and seamless.

Agile fits incomplete requirement nature of mobile projects: Mobile projects typically have deadlines and a quick turnaround time for market release. However, the initial requirements of mobile apps are generally incomplete, unclear, insufficient, uncertain, and change considerably during the development process. Developers will commonly put out an app with a limited set of features in the first release and update it in later versions. This nature of mobile app development fits with the iterative nature of Agile.

Agile development fits the experimentation \& adaptation of mobile apps: Mobile apps become better with each release. The process of refining and improving a mobile app is accomplished with the help of customer feedback. Agile gives early visibility to users and fits this necessary need for iteration.

Agile helps in identifying the risk in mobile projects at early stage: The release cycles for mobile app development projects are getting shorter and there is possibility of greater risks involved when dealing with tight releases. Small incremental releases made visible to the product owner and product team through its development help to identify any issues early in the project as they arise, making it much easier to respond to change. An adaptive approach and the clear visibility in Agile development adds value to the business by identifying risks and ensuring that any necessary decision can be taken at the earliest possible opportunity.

Agile is best suitable for quick delivery and short development lifecycle of mobile apps: Mobile applications are often characterized by an array of limitations such as rapid development and short development lifecycle. For initial release, a minimum viable product with prioritized features is built and delivered as fast as possible, followed by additional features in later versions. The bug-free quick delivery of product is well supported by Agile.

Mobile apps are downloaded and updated quickly, they need to seamlessly interact with back end servers whenever required which can be accomplished with numerous alterations and adjustments along with the way. Using Agile development approaches with sprints, enhanced quality assurance and multiple test cycles aids in successful development of mobile projects.

\subsection{Regarding an explanation on failed mobile projects using agile approach}

This section discusses organizations greatest concerns regarding adoption of Agile approaches for mobile projects, leading causes of failed mobile projects using Agile, and barriers to further adoption of Agile practices for mobile projects. 


\section{Organizations greatest concerns regarding the adoption of Agile for mobile projects}

Due to lack of documentation, loss of management control, regulatory compliance, inability to scale, lack of up front planning, management opposition, lack of predictability, development team opposed to change, lack of engineering discipline, reduced software quality, and quality of engineering talent (in descending order).

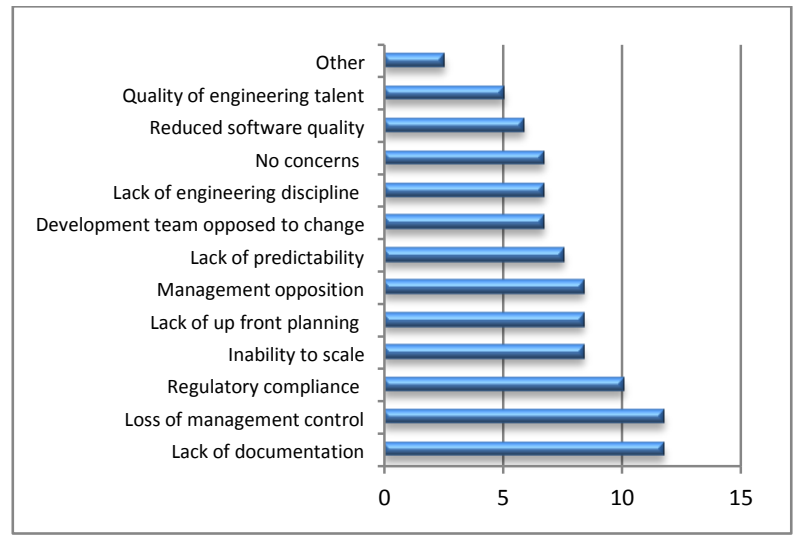

Figure 8: Organization's greatest concerns regarding the adoption of Agile development for mobile projects.

\section{Leading causes of failed mobile projects using Agile}

Due to company philosophy or culture at odds with core Agile values, lack of cultural transition, broader organizational or communications problems, lack of management support, external pressure to follow traditional waterfall processes, unwillingness of teams to follow Agile, lack of experience with Agile methods, insufficient technical training, and newness to Agile (in descending order).

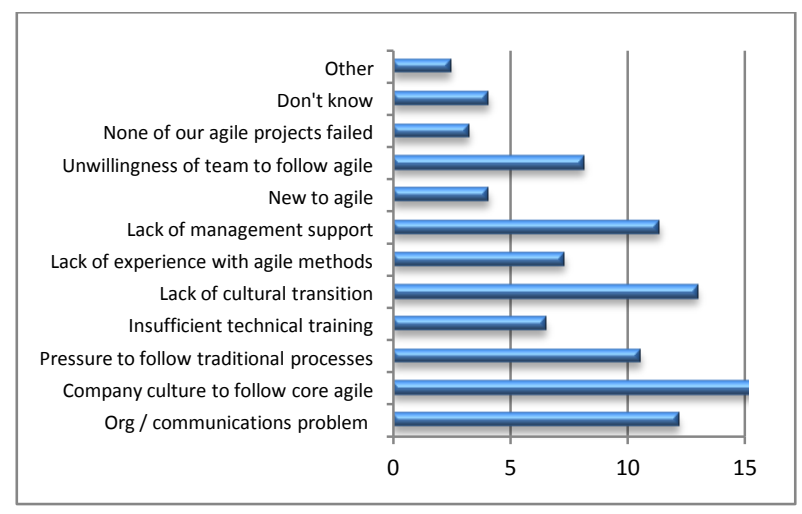

Figure 10: Leading causes of failed mobile projects using Agile techniques.

\section{Barriers to further adopt Agile in an organization for mobile projects}

Due to organization boundaries, customer collaboration, management support, general resistance to change, availability of personnel with right skills, trying to fit Agile elements into a non-Agile framework, project complexity, budget constraints, confidence in ability to scale, perceived time to transition, and personnel with Agile experience (in descending order).

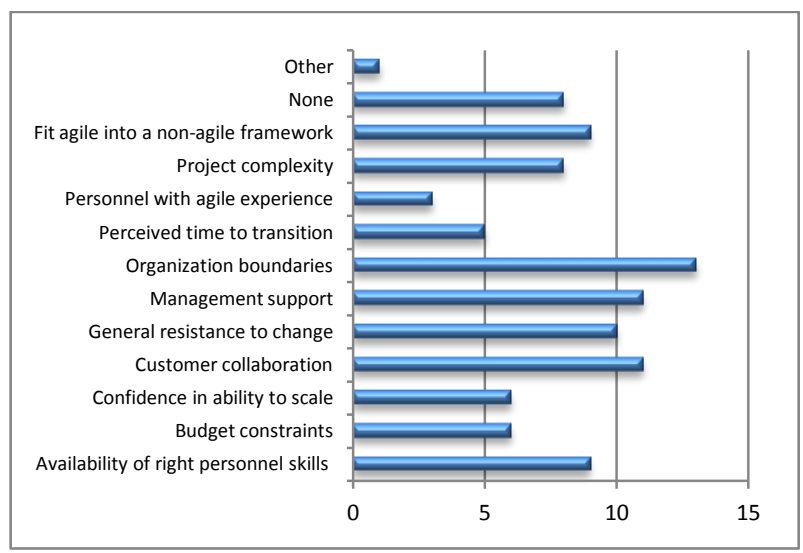

Figure 9: Barriers to further adopt Agile practices in your current organization for mobile projects.

\subsection{Regarding most appropriate Agile approach for mobile projects}

Regarding using a particular agile methodology for the development of mobile applications, many participants stated that there are many factors that influence the selection of a methodology and practices for a project. Moreover, it is very difficult, if not impossible to generalize and say that there is one methodology or set of practices that universally works best on all mobile app development projects.

There are many factors that can play out when determining a methodology and any Agile development process is suitable, as long as iterations (feedback loops) are kept short, the customer is involved and the team can adapt to changes in requirements and environment.

Agile is about people, not technologies and we should use what makes sense for our team and organization. The appropriateness of methods tends to depend more heavily on organizational context than on technology as platform rarely dictates methodology. There is the necessity and we should try to mix and match different methodologies considering that that we might not get it perfect in first try, just try again with lessons learned. Our focus should be on the principles of Agile, on Flow - doing one-thing-at-a-time - and Value, ensuring that the one-thing is the most valuable thing to do right now.

There are many Agile approaches such as XP, Scrum, Lean, Kanban, and DSDM, FDD and so on and they all share characteristics with the Agile Manifesto. The decision to adopt one methodology over the other depends on how well the team members know a particular methodology, how big the team is, and how the team is organized. To excel at Agile, everyone involved needs a solid understanding of the underlying Agile principles, and the organization needs to establish measurable goals for Agile. 
Most preferred Agile methodology for various practices involved during the development of mobile applications

As per survey participants, for developing mobile applications, the three most preferred methods for various Agile practices are - "For Engineering Practices": Scrum, XP, AM; "For Project Management Practices": Scrum, AM, AUP, DSDM; "For Accept Changes in iteration at any time": Scrum, Kanban, AM; "For Reduced Project Cost": Scrum, Lean, XP; "For Schedule Accomplishment": Scrum, AM, XP, FDD, AUP, Kanban; and "For Proper Resource Management": Scrum, Lean, AM, Kanban.

\section{Most widely used Agile methods for the development of mobile application}

The study shows the three most widely used Agile methods most useful for developing mobile applications are Scrum, $\mathrm{XP}$, and Lean software development methods. Below are the reasons stated by survey participants for choosing above mentioned Agile methods for the development of mobile application:

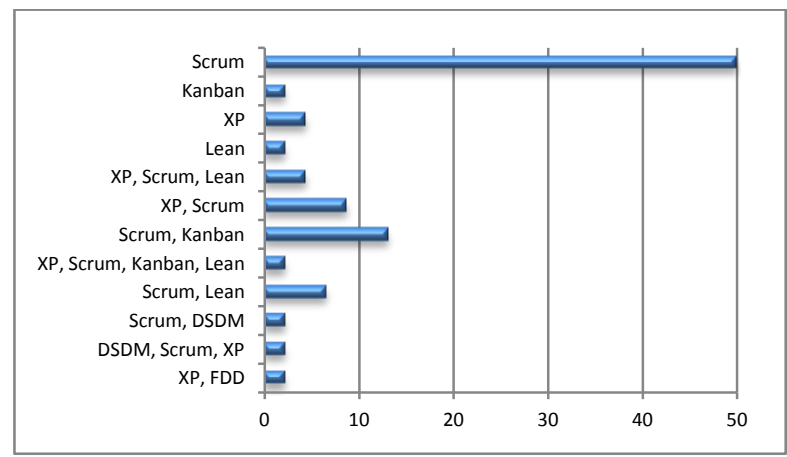

Figure 11: Most appropriate Agile approach for MAD.

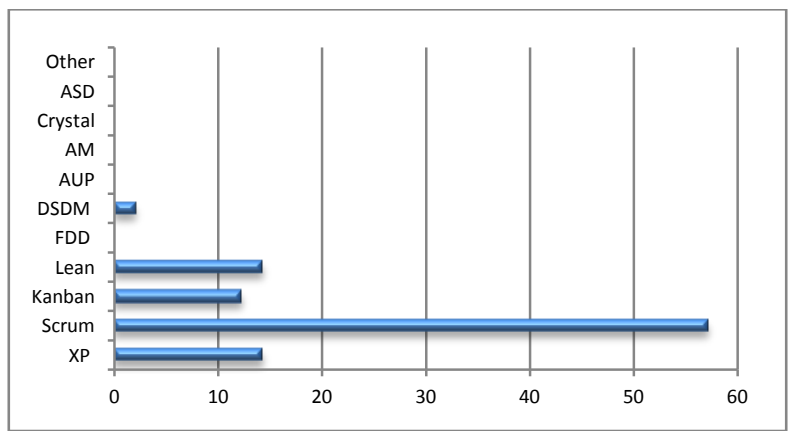

Figure 12: Agile approach most closely followed for MAD.

Scrum: As per majority of survey participants, Scrum is found to be the most effective mobile application because it offers daily stand-up meetings and provides all time project overview with details of impediments, to-do and completed task list. Scrum is time boxed, helps with estimation and is relatively light weight. It's also well known, easily understood and many people have done it before. Agile is about embracing changes and as Scrum is iteration based; it adopts changes quickly and has short turn around since mobile projects change so quickly. With shorter sprints, it provides quick feedback and more opportunities to re-build the app, helps to find the bugs easily at early stage, that way you can deliver value faster and also come up with changes in market technology.
- Daily stand-up meetings: This meeting is daily for maximum 15 minutes in which the team meets to update the task chart and report on progress and impediments.

- Short sprint/ iteration: A sprint (or iteration) is a "time boxed" effort. The duration is fixed in advance for each sprint and is normally between one week and a month.

Extreme Programming (XP): Extreme Programming is second preferred Agile approach that is actually practiced and developer participants believe that initial development is best with XP practices. XP is most suitable for the development of mobile application mainly due to behaviour-driven development (BDD), test-driven development (TDD) and continuous delivery (CI) because you won't be Agile for long without them.

Since Scrum does not say anything about engineering practices, so is usually supplemented by some XP core practices like TDD and CI that increases value delivery. TDD is important for designing before coding and test automation, very important when delivering every sprint and not wanting to break what was working in previous sprints and CI makes sure that nothing breaks.

- Pair Programming: XP Programmers write all production in pairs, two programmers working together at one machine. One is coding and thinking of the tactics of implementing the method, while the other is thinking about how the method strategically fits into other parts of the project.

- Test Driven Development: XP teams focus on validation of the software at all times. Programmers develop software by writing tests first that checks its correctness, and then software that fulfills the requirements reflected in the tests. Customers provide acceptance tests that enable them to be certain that the features they need are provided. Pair programming makes it easier for developers to adopt TDD.

- Continuous Integration: New features and changes are worked into the system immediately and XP teams focus on validation of software at all times. Each developer works on his own part of the project, and should integrate their code with other developer's code. Hence, continuous integration should be encouraged, which means to constantly integrate the code, at least once a day.

Lean Software Development: LSD is third preferred, to work on the product as the focus of Lean is continual learning and improvement along with reducing waste. LSD applies lean manufacturing principles to application development such as using the value stream concept, and leveraging tactics, sticking adhesive notes on a board to communicate the next needed task, much like Kanban cards is used in factories to manage materials. Therefore, lean software development principles enable to implement Scrum and Kanban principles effectively.

- Eliminate waste: Lean philosophy eliminate anything that does not add value to the customer as waste, such as, unnecessary code and functionality, delay in the software development process, unclear requirements, insufficient testing, bureaucracy and slow internal communication

- Continual learning \& improvement: The creation of software is continuous learning process with continual customer feedback. So the team as a whole is continually learning and improving throughout the delivery cycle. 


\section{DISCUSSION}

In literature review section, it was identified that an Agile approach is a natural fit for the development of mobile applications as traits observed in mobile software development maps perfectly to Agile themes. We discussed the main features and limitations of four methodologies (Mobile-D, MASAM, HME, and SLeSS) as proposed by researchers for the development of mobile applications using various Agile approaches. We also noticed during literature review that combination of some agile approaches, specially, Scrum, XP, Lean and Kanban has proven to be perfect and beneficial in the development of other software products. It was proposed that mixing of these approaches can be applied to mobile software projects also as no studies were found addressing combination of above mentioned Agile approaches. As predicated and anticipated, it was found from survey analysis outcomes that mobile community experts recommends integration of above mentioned Agile approaches for the development of mobile applications.

$\mathrm{XP}$ is based on four values - Communication, Feedback, Simplicity and Courage. Several rules and practices of Scrum support these values, such as user stories, iterations, stand-up meetings and more. But there are also some XP practices that have no similar corresponding match in Scrum, such as System Metaphors, Spikes, TDD and Continuous integration. Scrum and XP share many practices to support successful software development. The overlap is mainly in the planning part.

Although having many common practices and values, each of these disciplines has a different focus. Scrum follows project management practice and is more focused on managerial skills of both managers and developers. Thus, theoretically, scrum can be applied to any industry. XP on the other hand, is very specific to software development and includes many engineering practices, such as pair programming, continuous integration, test driven development. These practices make sure software quality is high. Scrum process means, we have a Scrum Master, we work in Sprints, we manage a project backlog and a Sprint backlog and we conduct daily scrums. Mixing it with XP means, we apply pair programming, CI, TDD and simple system design.

The main strengths of XP are fast development, cost saving, high satisfaction of client, test driven development resulting in less errors and acceptance of changing requirements. Scrum model is getting popularity from the last few years. Main strength of the Scrum model is high project management capability. It encourages us to embrace change. If our code is poorly written or we do not have an automated testing process, embracing change becomes a very difficult task. Scrum is a combination of generic project management practices and lacks system development life cycle (SDLC) phases about engineering of a software. For this purpose, practicing XP engineering practices is essential to support Scrum principles.

XP, Scrum and Lean models have various strengths and limitations. There is a desperate need of fusing the all three models to remove their shortcomings to solve the mobile industry problem in major.

Scrum and XP are two disciplines originating from the Agile manifesto that offers shorter development cycles, changing requirements, early customer feedback, rapid \& frequent delivery, and continuous improvement and working software which is ideal for mobile projects. On the other hand, Lean is adapted from Lean manufacturing of TOYOTA production system which focuses on the project management aspects by eliminating waste and by concentrating on continuous learning and improvement throughout the software lifecycle and specifies no technical practices and integrates well with other agile methodologies, such as XP.

The natural way to mix those together is to have a Scrum process with XP engineering along with lean principles and each of these disciplines can be used on its own and both have proved to be very effective. A mixture of Scrum and XP speeds up technology and business goals while developing new products and capabilities with great success. It enables the teams to be flexible and responsive to the product owner's requests while also following a process that allows them to estimate accurately and report out to the various stakeholders. Use of Lean is beneficial while they are in the supporting role for existing products.

This section discusses the validity of this study. First, the limitations and known challenges to the study are that responses were received only from people willing to be surveyed. Second, the instrument to measure Agile methods is untested and the statistical analysis may not be sensitive enough to measure greatly the variations in the data we collect, so its reliability cannot be fully determined in advance. Third, there is a risk of getting responses from people with strong feelings about the area, so there may be some bias towards the use of Agile methods. Fourth, the biases of the communities may be reflected in the results which mean that the survey sample may not represent the real world, and the result may be based on opinion and not fact. Fifth, the survey research may or may not be the best research method to analyse the impacts of Agile methods, in lieu of quantitative methods, which yield richer experiences.

\section{CONCLUSION}

Based on the literature study and our research findings, the study evaluates the use of Agile approach for success in mobile application development by determining the significance of the most used Agile engineering paradigms such as XP, Scrum, and Lean. Scrum is a product creation framework that follows project management practices and focuses on involving customers in the development process and delivering iteratively, XP focuses on all technical practices while lean software development principles focuses on reducing waste activities in the process.

This research has generated a number of contributions. The literature review identified suitable Agile practices for the development of mobile applications. The favourable evaluation of Agile methods by a large heterogeneous population of respondents suggests that Agile naturally fits mobile applications development needs. In addition, Agile methodologies was found to be most suitable for mobile development projects as they are short time, require flexibility, reduces waste and time to market.

However, it is imperative to be aware and take into account the different Agile approaches while developing mobile applications. This will make it possible to provide improvements to traditional software development and look into other types of software development where Agile may be beneficial to conducting software process improvements. 
The findings of the study show that adopting and adapting these Agile methods are highly suitable and proposed to help deliver enhanced speed and quality for mobile application development.

In conclusion, we strongly believe that this paper will provide relevant contribution that practitioners can benefit from our analysis in order to better understand the current trends in the mobile application development. We hope that the propositions formulated will offer guidance for future research.

\section{REFERENCES}

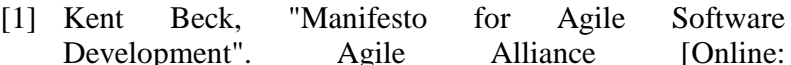
http://agilemanifesto.org] 2001.

[2] Harleen K. Flora, Xiaofeng Wang, Swati V. Chande. "An Investigation into Mobile Application Development Process, Challenges and Best Practices". International Journal of Modern Education and Computer Science (IJMECS). 2014

[3] Robert Holler. "Mobile Application Development: A Natural Fit with Agile Methodologies". Version One LLC. White Paper: [Online: www.versionone.com/pdf/mobiledevelopment.pdf] 2011.

[4] Nari Kannan. "Mobile development: Why using an Agile methodology makes sense". [Online: http://searchsoftwarequality.techtarget.com/tip/Mobiledevelopment-Why-using-an-Agile-methodology-makessense] 2011.

[5] Ben Morris. "A Software Engineering Model for Mobile App Development", [Online: http://static.bada.com/contents/blog/20110616/20introduction_to_bada_app03.pdf] 2011.

[6] Noor, "Is Agile approach a good fit for Mobile Application Development?” Nenu Tech. 2012.

[7] Chris Murphy, "Will Mobile Apps Drive IT Shops To Agile?" [Online: http://tech.newsgossipblog.com/willmobile-apps-drive-it-shops-to-Agile/. Information week] 2011.

[8] Steve Habermas, "Are Agile development \& mobile apps the perfect match?" [Online: http://www.verivo.com/mobility-watch/are-Agiledevelopment-mobile-apps-the-perfect-match/.Verivo Software] 2013.

[9] Scharff, C and Verma, R. "Scrum to Support Mobile Application Development Projects in a Just-in-time Learning Context", Workshop on Cooperative and
Human Aspects of Software Engineering (CHASE), pp. 25-31. 2010.

[10] Pekka Abrahamsson, "Keynote: Mobile software development - the business opportunity of today". Proceedings of the International Conference on Software Development. pp. 20-23. 2005.

[11] O. Salo and P. Abrahamsson, "Agile methods in European embedded software development organizations: a survey on the actual use and usefulness of extreme programming and scrum," Software, IET, vol. 2, pp. $58-64.2008$.

[12] Neek Alyani, Sara Shirzad, "Learning to Innovate in Distributed Mobile Application Development: Learning Episodes from Tehran and London", FedCSIS, pp. 497504. 2011.

[13] Anthony Wasserman, "Software Engineering Issues for Mobile Application Development". ACM. FoSER, 2010.

[14] Hang Su, "Some Management Principles Learned from Scrum Practices within a Global Software Development Project”, IACSIT. 2011.

[15] Scharff, C, "Guiding global software development projects using Scrum and Agile with quality assurance" Software Engineering Education and Training (CSEE\&T), 24th IEEE-CS Conference. 2011.

[16] Zaigham Mushtaq, "Novel Hybrid Model: Integrating Scrum and XP", International Journal of Information Technology and Computer Science (IJITCS). MECS. vol. 4, no. 6. 2012.

[17] Pekka Abrahamsson. "Mobile-D: An Agile Approach for Mobile Application Development," presented at csOOPSLA. Vancouver, Canada. 2004.

[18] V.Rahimian and R.Ramsin, "Designing an Agile methodology for mobile software development: A hybrid method engineering approach", Second International Conference on Research Challenges in Information Science, RCIS pp. 337-342. 2008.

[19] Yang-Jae Jeong, Ji-Hyeon Lee, and Gyu-Sang Shin, "Development Process of Mobile Application SW Based on Agile Methodology", 10th International Conference on Advanced Communication Technology, ICACT. pp. 362-366. 2008.

[20] Thiago Ferraz V. da Cunha, Valéria Lelli Leitão Dantas, Rossana M. C. Andrade: "SLeSS: A Scrum and Lean Six Sigma Integration Approach for the Development of Software Customization for Mobile Phones". SBES. 2011. 